Vol. 4 (5), pp. 135-138, June, 2014

ISSN: 2276-7762; ICV: 5.99

Copyright $@ 2014$, the copyright of this article is retained by the author(s)

DOI Link: http://doi.org/10.15580/GJBS.2014.5.033114169

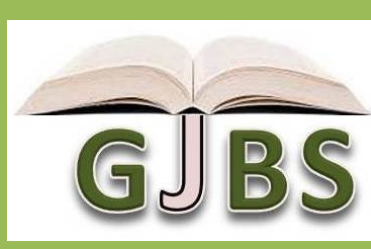

http://gjournals.org/GJBS

\title{
Prevalence of Gastrointestinal Helminthes in Stray Dogs of Tabriz City, Iran
}

\section{GAREDAGHI Yagoob ${ }^{1 *}$, SHABESTARI AsI Ali², AHMADI Seivan ${ }^{3}$}

\author{
1Department of Veterinary Parasitology, Collage of Veterinary Medicine, Tabriz Branch,Islamic \\ Azad University, Tabriz, Iran. \\ ${ }^{2}$ Department of Clinical Sciences, Collage of Veterinary Medicine, Tabriz branch, Islamic Azad \\ University, Tabriz, Iran. \\ ${ }^{3}$ Department of Veterinary Medicine, Collage of Veterinary Medicine, Tabriz branch, Islamic Azad \\ University, Tabriz, Iran.
}

Article No.: 033114169

Type: Research

DOI: 10.15580/GJBS.2014.5.033114169

Submitted: $31 / 03 / 2014$

Published: 28/06/2014

${ }^{*}$ Corresponding Author

Garedaghi Yagoob

E-mail: Y_garedaghi@yahoo.com,

Y_garedaghi@iaut.ac.ir

Keywords:

Stray dogs, Gastrointestinal

helminthes, Tabriz, Iran
Stray dogs are considered as an important health problem in societies because they can carry dangerous diseases such as hydatidosis, toxocariasis and coenurus cerebralis to humans and animals. Therefore, the study of helminthes infections in all parts of Iran for evaluation of infection state, and provision of the infection control programs is essential. The aim of this study was to determine the prevalence of parasitic helminthes in stray dogs around Tabriz city. In this study, 40 stray dogs were killed by shooting with the municipality officers and were dissected considering health rules. After laparotomy, internal organs are inspected, and bowels completely collected in separate containers containing $10 \%$ formaldehyde. Transferring the samples to the parasitological laboratory, the bowels were cut using forceps, and its contents passed through the sieve, and the worms were separated. Azo carmine \& carminic acid staining and lactophenol was used for diagnosis of isolated cestodes and nematodes. The number of $\mathbf{4 0}$ dogs, 34 dogs (85\%) had at least one species of cestodes and nematodes and acanthocephala that; $12.5 \%$ (5 dogs) infected by Echinococcus granulosus, $20 \%$ (8 dogs) by Taenia hydatigena, $22.5 \%$ (9 dogs) by Taenia multiceps, $17.5 / \%$ (7 dogs) by Dipylidium caninum and $52.5 \%$ (21 dogs) by Ancylostoma caninum, $45 \%$ (18 dogs) by Toxocara canis, 35\% (14 dogs) by Trichuris vulpis, $27.5 \%$ (11 dogs) by Macracanthorhynchus hirudinaceus. In this study, it was shown that helminthic rate in stray dogs is very high around Tabriz. These parasites are important in terms of health and economic aspects. Therefore, it is more essential that we note to parasitic infections, to develop control programs. 


\section{INTRODUCTION}

Dogs perform a range of cultural, social and economic functions in the society. They are kept as pets and companions, for hunting, as guards, draught animals, for food, or for commercial purposes (Tavakoli et al., 2002). Besides, some studies also suggest that keeping pets is associated with a higher level of self-esteem in children (Yang et al., 2009). Parasitism is the most commonly encountered disease in dogs all over the world. Regardless of the availability of medications to treat parasites, most parasites of dogs have highly evolved life cycle that make their elimination impossible. In addition, dogs are routinely affected without noticeable infestation. A dog can have internal parasites even though the fecal sample test is negative (Heidari et al., 2011). Pervious study has indicated that parasitic diseases in general and gastro-intestinal helminthes and protozoan in particular have been identified as the major impediment to dog health worldwide owing to the direct and indirect losses they cause (Wachira et al., 1994). Most of the parasites affect the dogs sub-clinically. Consequently, dogs may harbor a wide range of parasites with zoonotic potential causing health risks to human beings (Wachira et al., 1993). Some of the parasites like $E$. granulosus involve food animals as an intermediate host and cause great economic loss through organ condemnation at the level of slaughterhouse (Walters and Craig, 1992). Gastrointestinal helminthes of dogs pose serious impact both on the host and human beings. It impede the successful rearing of dogs and result in losses that are manifested by lowered resistance to infectious diseases, retarded growth, reduced work and feed efficiency and general ill. Parasitized animals show a variety of signs depending on the parasite species (Walters, 1977). These signs are attributed to intestinal obstruction, irritation, mal-digestion, mal-absorption and protein losing induced by the parasites (Ajlouni et al., 1984). In areas of high population such as urban and peri-urban, dog keeping could be a risk to the transmission of zoonoses, some of which could be of parasitic origin (Razmi et al., 2006). The prevalence of dog helminthes considerably varies (Fallah et al., 1995) from one region to another and among the different diagnostic techniques employed. In Iran, however, very little attention was given for parasites of dogs. As a result, the works done on the prevalence of the different gastrointestinal parasites of dogs so far are scanty (Arbabi et al., 2004; Hosseini et al., 1998; Abdi, 2003). The aim of this study was to determine the prevalence of parasitic helminthes in stray dogs around Tabriz city.

\section{MATERIALS AND METHODS}

In this descriptive study, samples prepared from the carcasses of stray dogs were killed by shooting with the municipality officers. From February 2013 to February 2014, a total of 40 dogs (20 male dogs and 20 female dogs in 1-4 years old) were dissected and examined macroscopic and microscopic. After physical examination, to determine the age and sex, the dog's carcass in the supine position from end sections of ribs longitudinal and perpendicular was slit with the scalpel. Early the mesenteric, and then the beginning of the stomach to the end of anus were removed.

To prevent removal of intestinal contents and spread of the infection thread, bowel ends were closed and transferred to the laboratory. Intestines were split in enamel basin with splitter scissors and its contents were washed with mild stream of water and passed thorough sieves containing $1 \mathrm{~mm}$ pores. Helminthic samples were collected in glass containers containing $10 \%$ formalin for staining for the final diagnosis. Thus, the lactophenol and azo carmine was used for temporary staining and carminic acid \& alcohols 50, 70, 80, 90, and $100 \%$ for permanent staining. The samples were carefully evaluated using a microscope. The keys in the Schmidt's book were the reference for identifying gender and species of cestodes and nematodes.

\section{RESULTS}

Four species of cestodes and four species of nematodes were isolated from a total of 40 dogs. Of 40 dogs, 34 dogs $(85 \%)$ had at least one helminthic parasite species, including 5 dogs affected by Echinococcus granulosus, 8 dogs by Taenia hydatigena, 9 dogs by Taenia multiceps, 7 dogs by Dipylidium caninum and 21 dogs by Ancylostoma caninum, 18 dogs by Toxocara canis, 14 dogs by Trichuris vulpis, 11 dogs by Macracanthorhynchus hirudinaceus. Echinococcus granulosus, Taenia multiceps, Dipylidium caninum, Taenia hydatigena and Ancylostoma caninum, Toxocara canis, Macracanthorhynchus hirudinaceus are pathogen in humans and they have health importance. The highest rate of infection associated with Ancylostoma caninum and the lowest rate with $E$. granulosus (Table 1). There was no significant difference in the prevalence between male and female dogs and between different age groups with T-test and chi-square ( $p>0.05)$. 
Table 1: The prevalence of cestodes and nematodes in stray dogs around Tabriz, Iran

\begin{tabular}{|l|c|c|}
\hline \multirow{2}{*}{ Helminthes } & \multicolumn{2}{|c|}{ Infection Rate } \\
\cline { 2 - 3 } & 18 & Percent \\
\hline Toxocara canis & 21 & 45 \\
\hline Ancylostoma caninum & 14 & 52.5 \\
\hline Trichuris vulpis & 11 & 35 \\
\hline $\begin{array}{l}\text { Macracanthorhynchus } \\
\text { hirudinaceus }\end{array}$ & 7 & 27.5 \\
\hline Dipylidium caninum & 5 & 17.5 \\
\hline Echinococcus granulosus & 9 & 12.5 \\
\hline Taenia multiceps & 8 & 22.5 \\
\hline Taenia hydatigena & & 20 \\
\hline
\end{tabular}

\section{DISCUSSION}

Most intestinal parasites detected in this study, have a worldwide distribution. Although, incidence and prevalence of each parasite, in the larval and adult stages, respectively, in humans and animals is different in various regions based on knowledge, cultural practices, climatic conditions, and diagnostic possibilities. Stray dogs have not the owner, so they had not taken any anti-parasitic drug. Given the uncertain sources of their nutrition, intestinal infections in these dogs are natural. Echinococcus granulosus is one of the most important cestodes that are reported in this study. Although the prevalence of this parasite in Tabriz city is lower than other studies in Iran, because it has multiple interface hosts, and affects a variety of host organs, thus, the presence of the disease in the region is a potential risk; More accurate and comprehensive studies should be implemented to obtain the overall prevalence of the disease through studying on infection rate of slaughter animals, seroepidemiological and surgical assessments of hydatid cyst in treatment centers. Probably, due to drought in recent years that it is redounded to selling cattle by ranchers, may be the cause of the low prevalence of E. granulosus in stray dogs around Tabriz. The Taenia multiceps infection in this study reported; it's the cause of a serious disease called coenurus cerebralis. Coenurus cerebralis is a specific cerebral disease in sheep, but few human cases have been reported in the world. This shows that the disease is common between humans and animals and may be transmitted to humans.

Dipylidium caninum is reported in Tabriz dogs. This parasite cause human's infection rarely, but is classified as a zoonosis, that is a health problem. Ancylostoma caninum infection is the most common nematodes among dogs in this area, thus, there has been usually the Hook-worm disease in the Human of this region. Bahrami et al. studied on intestinal parasites in stray dogs in llam using fecal analysis (rather than dissection in the present study); they reported the prevalence of Taenia taeniaformis 19.64 percent and the prevalence of Echinococcus species 6.25 percent. But all Taenia's eggs are quite similar to each other, and they cannot be used to differentiate Taenias; scolexes \& segments are used for this reason that it is possible only with dissection (Bokaei and Moazzeni, 2008; Bahrami et al., 2011). In a study for helminthic parasites in stray dogs in Isfahan city at $2011,60 \%$ of dogs were infected; and seven species of cestodes including Taenia ovis $24 \%$, Taenia hydatigena $13.54 \%$, Taenia pisiformis $5.21 \%$, Taenia multiceps $5 \%$, Echinococcus granulosus $27 \%$, Dipylidium caninum 22\%, and Mesocestoides lineatus 5 percent have been reported (Pestechian et al., 2011). Taenia hydatigena infection rate in this study was similar to that of Pestechian's study (Yusefi, 2011); this parasite can also cause significant damage to the economy. Macracanthorhynchus hirudinaceus has been reported in a variety of studies; and there are evidences about transmission of the parasite to humans that may cause diarrhea. It increases the importance of the parasite prevalence. Helminthic infection rate in dogs of Hamadan city was $100 \%$, whereas, the prevalence of $E$. granulosus 48.3 percent; it is much higher than our study (Fallah et al., 1995). Some studies have been conducted in different parts of the world, too. For example, the overall prevalence in Brazilian stray dogs has been reported 54.33 percent (Katagiri and Oliveira-Sequeira, 2008). In Kermanshah Province, the overall rate of infection was equivalent to that which has been reported 82.5\% (Rokni, 2009; Abdi1 et al., 2013). There is no doubt that presence of millions of dogs in our country, under any circumstances cannot be justified and should be planned in a systematic attempt to destroy them. 
Comparison of the infection rate in different areas may represent a positive or negative role of the municipalities in obliterating of the stray dogs (Garedaghi and Safarmashaei, 2011). Besides, the better supervision of health systems, and even the role of the veterinary inspection on meat and slaughterhouse waste, is related to the results. Meanwhile, the changes in recent climatic states like rainfall decline and drought should be considered; and role of cities development \& urbanism, interfere to natural reservoirs privacy of this parasites should be lionized.

\section{ACKNOWLEDGEMENTS}

The authors would like to thanks Tabriz Branch, Islamic Azad University for the financial supports of this research, which is based on a research project contract.The authors declare that they have no conflicts of interest.

\section{REFERENCES}

Abdi J: Study of Cestodes in Stray Dogs in Isfahan and its Hygienic Importance. Isfahan: Isfahan university of Medical sciences; 2003: 50-54.

Abdi1 J., Asadolahi Kh., Maleki Mh., Ashrafi Hafez A. Prevalence of Helminthes Infection of Stray Dogs in Ilam Province. Journal of Paramedical Sciences, $2013, .4(2): 47-50$.

Ajlouni AQ, Saliba EK, Disi AM :Intestinal cestodes of stray dogs in Jordan. Zeitschrift fur Parasitenkunde 1984, 70(2):203-210.

Arbabi M, Doroudgar A, Hooshyar H, Mobedi I: A survey of cestode infections of carnivores in the Kashan region. Journal of the Faculty of Veterinary Medicine, University of Tehran 2004, 59(3):289-293.

Bahrami A, Doosti A, Nahravanian H, Noorian A, Ahmadi S: Epidemiological Survey of Gastro-Intestinal Parasites in Stray Dogs and Cats Australian Journal of Basic and Applied Sciences 2011, 9(5):19441948.

Bokaei S, Moazzeni M, Drodgar M: Study of intestinal worms of pet dog in Shiraz. Veterinary Journal of Tehran University 2008, 3(63):169-164.

Fallah M, Taherkhani $\mathrm{H}$, Sadjjadi M: Echinococcosis in stray dogs in Hamedan, west of Iran. Iranian J Med Sci 1995, 29:170-172.

Garedaghi Y. Safarmashaei S. Prevalence of gastrointestinal helminthic infestation in pet and stray dogs in Tabriz, Iran. Journal of animal and veterinary advances, 2011, 10(11):1477-1479.

Heidari Z, Mohebali M, Zarei Z, Aryayipour M, Eshraghian MR, Kia EB, Shodajei S, Abdi J, Rakhshanpour A, Rokni MB: Seroepidemiological study of human hydatidosis in meshkinshahr district, ardabil province, Iran. Iranian Journal of Parasitology 2011, 6(3):19-25.

Hosseini SH, Bokaie S, Motevaselolhoseini M: Hydatid cyst and its role in epidemiology of Echinococcus granulosus in camel. Journal of theFaculty of Veterinary Medicine, University of Tehran 1998, 53(3/4):83-86.

Katagiri S, Oliveira-Sequeira TC: Prevalence of dog intestinal parasites and risk perception of zoonotic infection by dog owners in Sao Paulo State, Brazil. Zoonosis Public Health 2008, 55(8-10):406-413.

Pestechian N, Rasuli A, Yusefi H: Intestinal helminth parasites of Isfahan. Journal of Isfahan Medical School 2011, 29:173-179.

Razmi GR, Sardari K, Kamrani AR: Prevalence of Echinococcus granulosus and other intestinal helminths of stray dogs in Mashhad area, Iran. Archives of Razi Institute 2006, 61(3):143-148.

Rokni MB: Echinococcosis /hydatidosis in Iran. Iranian $J$ Parasitol 2009, 4(1):1-16.

Tavakoli HR, Bahonar AR, Jonidi NA: Epidemiology of hydatidosis in Iran during 2002-2006. Iranian Journal of Infectious Diseases and Tropical Medicine 2008, 13(42):67-71.

Wachira TM, Sattran M, Zeyhle E, Njenga M: Abattoirs and echinococcosis in Nairobi dogs. Transactions of the Royal Society of Tropical Medicine and Hygiene 1994, 88(2):166-169.

Wachira TM, Sattran M, Zeyhle E, Njenga MK: Intestinal helminths of public health importance in dogs in Nairobi. East African Medical Journal 1993, 70(10):617-619.

Walters TM, Craig PS: Diagnosis of Echinococcus granulosus infection in dogs. Vet Rec 1992, 131(2):39-40.

Walters TMH: Hydatid disease in Wales. Transactions of the Royal Society of Tropical Medicine and Hygiene 1977, 71(2):105-108.

Yang YR, McManus DP, Huang Y, Heath DD: Echinococcus granulosus Infection and Options for Control of Cystic Echinococcosis in Tibetan Communities of Western Sichuan Province, China. PLoS Negl Trop Dis 2009, 3(4):426-429. 